\title{
Producción científica de los docentes de cursos de investigación en facultades de medicina de Latinoamérica: ¿se está dando ejemplo?
}

\author{
Álvaro Taype-Rondán, Américo Peña-Oscuvilca, Alfonso J. Rodríguez-Morales
}

Cada vez se habla más de la importancia de la investigación científica en el pregrado de medicina [1-3], en favor de animar a un número cada vez mayor de estudiantes a inclinarse en un futuro hacia una especialidad en investigación o a desarrollar actividades de investigación en cualquier especialidad. Esto aumentaría la cantidad y calidad de la investigación, que constituye una herramienta valiosa para mejorar la salud de la población [4].

A pesar de la importancia que tiene en la actualidad la investigación estudiantil, existen reconocidas dificultades para llevar a cabo dicha actividad [5]. En Latinoamérica, es común escuchar que muchos estudiantes manifiestan que los cursos de investigación científica en sus facultades (metodología de la investigación, bioestadística, redacción científica) son tediosos y llegan a desanimar a aquellos que tienen pensado dedicarse a la investigación [6,7]. Esta situación es preocupante y podría estar limitando la investigación estudiantil en dicha región y el interés de los futuros médicos en el tema.

Es probable que en numerosas universidades el problema radique en el escaso compromiso de los docentes con la investigación estudiantil y en su escasa experiencia en investigación científica. En ambos casos, los estudiantes carecerían de una adecuada asesoría, lo que dificultaría su quehacer científico. Por ello, quienes son los responsables de dirigir los cursos de investigación de pregrado deberían ser profesionales que acrediten investigaciones demostrables por sus publicaciones científicas y no sólo por la posesión de títulos o grados universitarios.

Se considera que publicar artículos en revistas científicas indizadas en bases de datos de impacto medio-alto (SciELO, Scopus, Medline, Science Citation Index) debe constituir el principal elemento para ser considerado investigador, toda vez que es con la publicación con la que se termina el proceso de una investigación científica. En este contexto, denominar a un investigador -más aún si es docente- de tal forma sin que existan publicaciones cien- tíficas en su haber resulta tan inaceptable hoy día como un cirujano instruyendo en cirugía sin tener un número acreditado de intervenciones realizadas que puedan dar cuenta de su verdadera experiencia en su ámbito, más allá de poseer el debido título en el área.

De esta manera, las escuelas de medicina latinoamericanas que se interesen por mejorar las competencias de sus estudiantes en investigación deberían preocuparse por capacitar a los docentes en redacción y publicación científica (actualmente numerosas sociedades científicas de estudiantes y profesionales imparten talleres y cursos al respecto), evaluar la producción científica entre los docentes de los cursos de investigación, desarrollar programas de estímulos (incluidos los económicos) a los docentes que mantienen una buena producción científica -y más aún cuando lo hagan con estudiantes de medicina- y asumir la producción científica estudiantil (traducida como publicación de artículos científicos) como el principal indicador de los cursos de investigación (todos los cursos deberían aportar contribuciones científicas como consecuencia de su actividad) [1-3].

Estas estrategias pueden implementarse con mayor o menor rapidez en las escuelas de medicina de Latinoamérica. Sin embargo, creemos necesario hacer un llamamiento a los estudiantes, a los docentes, a las autoridades universitarias y a los acreditadores de facultades de medicina, quienes deben trabajar en conjunto para promover y cuantificar la publicación científica entre los docentes de los cursos de investigación y los estudiantes de medicina.

\section{Bibliografía}

1. Oróstegui-Pinilla D, Cabrera-Samith I, Angulo-Bazán Y, Mayta-Tristán P, Rodríguez-Morales AJ. Encuentros internacionales de revistas científicas estudiantiles de las ciencias de la salud en Latinoamérica, 1998-2008. Rev Panam Salud Publica 2009; 25: 469-70.

2. Cabrera-Samith I, Oróstegui-Pinilla D, Angulo-Bazán Y,
Sociedad Científica de Estudiantes de Medicina; Universidad de San Martín de Porres; Lima, Perú (A. Taype-Rondán). Facultad de Medicina Humana; Universidad Nacional José Faustino Sánchez Carrión; Huacho, Perú (A. PeñaOscuvilca). Facultad de Ciencias de la Salud; Universidad Tecnológica de Pereira y Oficina de Investigación Científica; Cooperativa de Entidades de Salud de Risaralda, COODESURIS; Pereira, Colombia (A.J. RodríguezMorales).

Correspondencia:

Dr. Alfonso J. Rodríguez Morales. Oficina de Investigación Científica. Cooperativa de Entidades de Salud de Risaralda (COODESURIS).

Avda. 30 de Agosto, n. ${ }^{\circ} 87-298$

Comuna Olímpica. Pereira 660001 . Risaralda, Colombia.

E-mail:

arodriguezm@utp.edu.co

(c) 2013 FEM 
Mayta-Tristán P, Rodríguez-Morales AJ. Revistas científicas de estudiantes de medicina en Latinoamérica. Rev Med Chil 2010; 138: 1451-5

3. Pereyra-Elías R, Rodríguez-Morales AJ, Mayta-Tristán P. Undergraduate publication in Latin America: role of medical students' scientific societies. Med Teach 2011; 33: 594

4. Ley TJ, Rosenberg LE. The physician-scientist career pipeline in 2005: build it, and they will come. JAMA 2005; 294: 1343-51.

5. Escudero-Sepúlveda A, Escudero-Sepúlveda J, Rodríguez-
Morales AJ. La redacción de un trabajo científico. Invest Clin 2012; 53: 111-2.

6. Molina-Ordóñez J, Huamaní C, Mayta-Tristán P. Apreciación estudiantil sobre la capacitación universitaria en investigación: estudio preliminar. Rev Peru Med Exp Salud Publica 2008; 25: 325-9.

7. Ángel-Isaza AM, Botero-Suárez HF, González DC, Ospina LP, Velasco MM, Ocampo MF. Interés de los estudiantes de medicina por la investigación. CIMEL 2010; 15: 9-13. 